Metals and Ceramics Division

CRADA Final Report

For CRADA Number ORNL 97-0477

Atomic Scale Structure of Ultrathin Magnetic Multilayers and Correlation with Resistance and Giant Magnetoresistance and Spin-Dependent Tunneling

W. H. Butler

Oak Ridge National Laboratory

G. E. Ice

M. K. Miller

S. J. Pennycook

E. Voelkl

J. M. Daughton

Nonvolatile Electronics, Inc.

H. Kaakani

Honeywell Solid State Electronics Center

Date Published - February 2001

Prepared by the OAK RIDGE NATIONAL LABORATORY

Oak Ridge, TN 37831

managed by

UT-BATTELLE, LLC

for the

U.S. Department of Energy

Under contract DE-AC05-00OR22725

APPROVED FOR PUBLIC RELEASE

UNLIMITED DISTRIBUTION 
Metals and Ceramics Division

CRADA FINAL REPORT

FOR

CRADA NO. ORNL 97-0477

\title{
Atomic Scale Structure of Ultrathin Magnetic Multilayers and Correlation with Resistance and Giant Magnetoresistance and Spin-Dependent Tunneling
}

\author{
W. H. Butler \\ Oak Ridge National Laboratory \\ G. E. Ice \\ M. K. Miller \\ S. J. Pennycook \\ E. Voelk1 \\ J. M. Daughton \\ Nonvolatile Electronics, Inc. \\ H. Kaakani \\ Honeywell Solid State Electronics Center \\ Date Published - February 2001 \\ Prepared by the \\ OAK RIDGE NATIONAL LABORATORY \\ Oak Ridge, TN 37831 \\ managed by \\ UT-BATTELLE, LLC \\ for the \\ U.S. Department of Energy \\ Under contract DE-AC05-00OR22725
}

Approved for Public Release

Unlimited Distribution

*This work was supported through a CRADA with Honeywell International, Inc., Plymouth, MN and NVE, Eden Prairie, MN, sponsored by the Laboratory Technology Research Program, Office of Science, US. Department of Energy, under contract DE-AC05-00OR22725 with Oak Ridge National Laboratory, managed by UT-Battelle, LLC. 


\title{
CRADA Final Report
}

ORNL97-0477

\author{
Honeywell Solid State Electronics Center \\ And \\ NVE Corporation (formerly Nonvolatile Electronics, Inc.)
}

\begin{abstract}
ORNL's advanced characterization capabilities were used to determine the physical and chemical structure of magnetic multilayer films intended for application in non-volatile magnetic random access memory devices and as magnetic sensors. ORNL modeling capabilities were used to incorporate this information into a first-principles based tool that can be used to model the magnetic and transport properties of these films. This modeling capability should be useful for understanding and optimizing novel magnetoelectronic devices.
\end{abstract}

\section{Objectives}

The objectives of this CRADA were:

1. To determine the physical, chemical and magnetic structure of GMR films on length scales relevant to GMR, i.e. from $2 \AA$ to $200 \AA$.

2. To relate this structure to the electrical resistivity of the films by developing a theory of transport capable of treating the types of disorder observed.

3. To develop a sufficiently detailed understanding of the connections between processing parameters, structure, and transport that we can modify the processing parameters to optimize films for GMR and also use resistivity as an in-situ, real-time monitor of film quality.

\section{Benefits to the Funding DOE Office's Mission}

An important priority of the Department of Energy's strategic goals is to ensure that the United States maintains its leadership in science and technology. The Laboratory Technology Research (LTR) program is the lead program in the Department of Energy's Office of Science for leveraging science and technology to advance understanding and our country's economic competitiveness through cost-shared, research collaborations with private sector partners. This research program provided important understanding of the physical chemical and magnetic structure of magnetic multilayer films and showed how these structures impact the giant magnetoresistance effect (GMR). GMR forms the basis for an important new technology, nonvolatile magnetic random access memory. 


\section{Technical Discussion of Work Performed}

\section{Giant Magnetoresistance (GMR) and Magnetic Random Access Memory (MRAM)}

The GMR effect is the change in electrical resistance of a magnetically inhomogeneous conductor that occurs when an applied magnetic field aligns the magnetic moments in different regions. For applications to magnetic random access memory (MRAM) the inhomogeneous conductors typically consist of two magnetic layers separated by a non-magnetic spacer layer. The two magnetic layers change their relative orientation from anti-parallel to parallel upon application of an external magnetic field. The two different relative orientations result in different resistances of the film. For GMR-MRAM applications, the two magnetic layers usually have different thicknesses. The orientation of the moments in the thinner layer can be switched in smaller fields than those of the thicker layer. This allows the thinner layer to be used as a "read" or "sense" layer while the thicker layer is used to store the information. Reading is accomplished by applying small magnetic fields that turn the moments of the thinner layer but not the thick layer. From the sense of the change in resistance one can determine the orientation of the moments in the thicker layer and the stored information. Writing is accomplished by applying a field large enough to switch the direction of the moments of the thicker layer. Because of the shape of the magnetic memory cell, the moments are stable in two opposite orientations. The magnetic fields for sensing and writing are obtained from currents flowing through a crossed array of "word" and sense lines.

The change in resistance due to the change in relative orientation of the magnetic moments of the films (GMR is often expressed as the ratio, $\Delta \mathrm{R} / \mathrm{R}$ ) and the strength of the magnetic fields at which the films change their direction (switching fields) are very important parameters determining the performance of GMR based memories. These parameters are very strongly dependent upon the structure of all layers, their chemical composition, the quality of their depositions and most significantly the quality of the interfaces between all of the layers involved.

\section{Film Characterization}

Because the viability of the GMR-MRAM technology depends critically on obtaining relatively large values of $\Delta R / R$ for relatively small fields (and hence relatively small currents), and because both the GMR $(\Delta \mathrm{R} / \mathrm{R})$, and the switching fields depend sensitively on the structure of the films we utilized the advanced characterization techniques at ORNL to determine film structure.

A variety of films fabricated using different deposition and processing conditions, were studied to determine the extent of impurity incorporation, to investigate the level of interface roughness, and to determine their grain size. These ORNL studies utilized several x-ray spectroscopies including X-ray Diffraction (XRD), X-ray Photoelectron Spectroscopy (XPS), and X-ray Fluorescence Spectroscopy (XFS). Transmission Electron Microscopy (TEM), Scanning Transmission Electron Microscopy (STEM), and electron holography were also used at ORNL to study the films.

Magnetic properties of single magnetic nanolayers, GMR stacks and patterned memory bits were investigated at Honeywell using both B-H looper and Alternating Gradient Magnetometry (AGM). These studies were supplemented by investigations at 
ORNL using SQUID magnetometery. A major effort was made to apply 3D Atom Probe Field-Ion Microscopy (3DAP-FIM) to determine film and interface structure with atomic scale resolution

Detailed structural studies of Tantalum seed layers and their effect on magnetic nanofilms were performed. A series of experiments involving single magnetic permalloy nanostructures grown on tantalum were performed in a collaborative effort to determine the thickness of magnetically dead layers at a variety of interfaces. These studies led to changes in design to reduce the structural and magnetic roughness by placing interdiffusion barriers and smoothing layers at crucial interfaces in the GMR stacks.

\section{Magnetic and Transport Modeling}

The understanding of the physical chemical and magnetic structure obtained from our characterization of the films was used to develop models for the magnetic coupling and for the resistance and magnetoresistance of the films. The model for the magnetic coupling extended Nèel's theory of magnetic coupling due to conformal large scale interfacial roughness. The model for electron transport utilized a first-principles calculation of the spin-dependent electronic structure (Fermi surface and electron velocities) of each of the active layers. We also calculated the spin-dependent transmission and reflection coefficients between each of the active layer materials. These calculated quantities form the basis for calculating the resistance, magneto-resistance and current distribution through the film. The calculation is performed using the semiclassical Boltzmann equation. Both the magnetic coupling and the transport codes have been made accessible to our industrial partners.

\section{Inventions (Made or Reported)}

No inventions were disclosed.

\section{Commercialization Possibilities}

Honeywell has recently sold its rights to commercial application of GMR-MRAM technology. Honeywell continues to work on defense and aerospace applications of the technology. NVE Corporation sells several products based on GMR technology including magnetic sensors and isolators. Our transport codes are expected to have wide application to design of magnetic memory devices as well as to design of read heads for disk drives and sensors.

\section{Plans for Future Collaboration}

Plans for future collaboration are under discussion both with Honeywell and with NVE.

\section{Conclusions}

ORNL characterization and modeling capabilities are a vital asset for understanding magnetic multilayer materials and for the design of magnetic memory and read sensors. 


\section{DISTRIBUTION FOR ER-LTR CRADAS}

\section{Internal Distribution}

W. H. Butler, 4500S, 6114

P. A. Carpenter, 4500 N, 6269

L. L. Horton, 4500S, 6132

G. E. Ice, $4500 S, 6118$

M. K. Miller, 4500S, 6136

T. L. Payne, 111 UNV, 6499

S. J. Pennycook, 3025M, 6030

T. M. Rosseel, 4500S, 6138

E. Voelkl, 4515, 6064

K. M. Wilson, 111-B UNV, 6499

Office of Technical Information and Classification, 4500N, 6254

\section{External Distribution}

J. M. Daughton, NVE, 11409 Valley View Road, Eden Prairie, MN 55344-3617

H. Kaakani, Honeywell International, Inc., 2001 Highway 55, Plymouth, MN 55441

DOE-WFO, MS G209

S. J. Barish, Department of Energy, SC-32, 19901 Germantown Rd., Germantown, MD 20874-1290

W. M. Polansky, Department of Energy, SC-32, 19901 Germantown Rd., Germantown, MD 20874-1290 


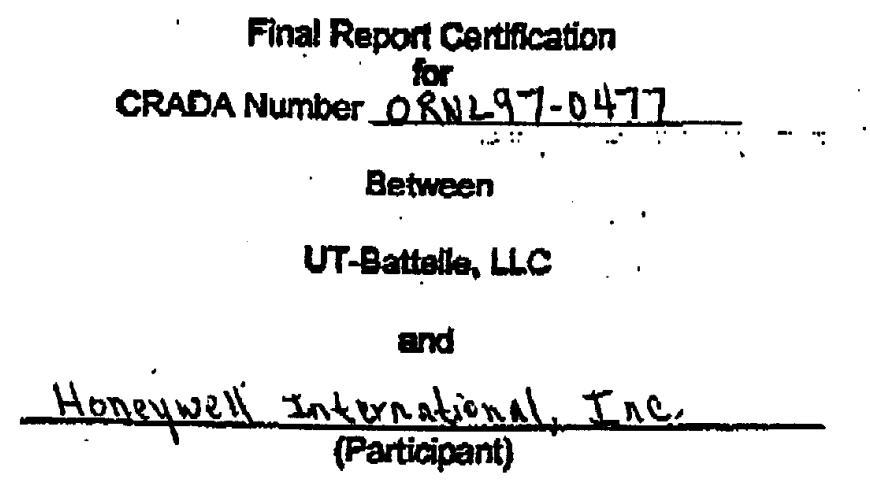

Instructions:

Mark the appropriate statenent in fe or to below with an 'DX." Reter to the articles in the CRAOA terms and conditions qoverning the identilication and makhng of Protected CRADA information.

If no PCl is identitiod, the report will be distributed without restrietion. If PCI is identified, the report distribusion will be finited in accondance with the CRADA terme and conditions governing release of data. In all cases ltems 2 and 3 gust be true. That $k$, the report cannot contain Proprletary intormation and a disclosure mesat be filed proor to release of the report.

This certification may either be made by using this form or may bo made on company letterhead if the Particioanl desines. A faxed coov of this cortupleted form is acceotable.

The following certification is made for the subject final report:

1. (a) The final report contains information that qualities as "Protected CRADA Information" (PC). The PCl legend is printed on the report cover, and Tor the PCl is clearly identified.

(b) The final report does not contein "Protected CRADA Intormation." The "Approved for Public Release" legend is printed on the repont cover.

2. The final repon does not contain Proprietary Infomation.

3. By the signature below, the Participant has no objection to the public distribution of the final report due to palentable information.

For the Partipinant - HASSAN KAAKANI]

Pregram \& Businés Developwent Manager

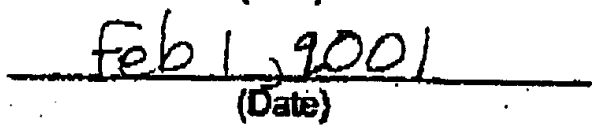




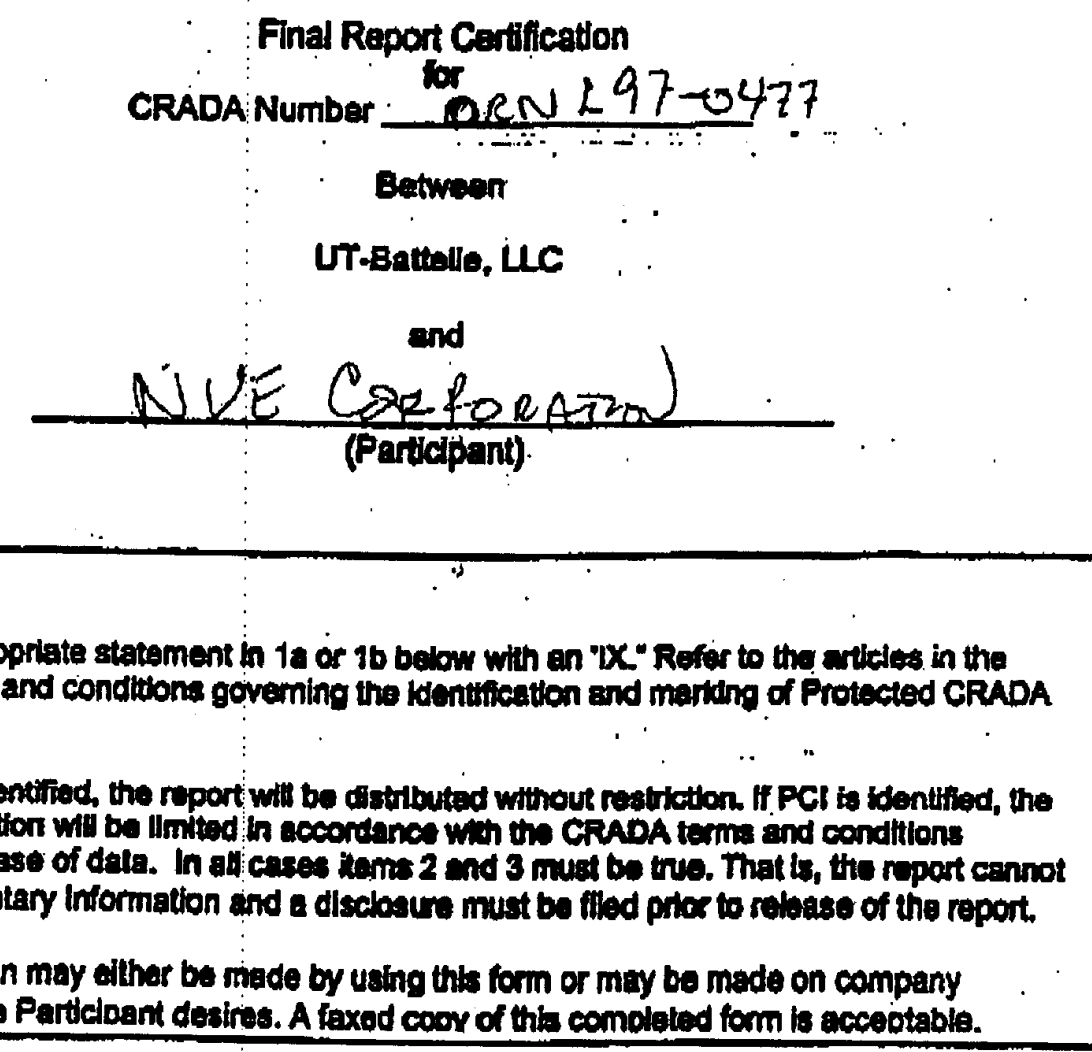

The following certification is mode for the subjoct final report:

1. (a) The final report contains information that qualifies as Protected CRADA Information" (PCI). The PCl legend la printed on the report cover, and OR the $\mathrm{PCl}$ is clearty Identified.

(b) The thal report does not contaln "Protected CRADA Information." The "Approved for Public Release" logend it pinted on the report cover.

2. The final report does not contain Propriotiary Intormation.

3. By the signature below, the Participant has no objection to the public distribution of the final report dus to patentable information.

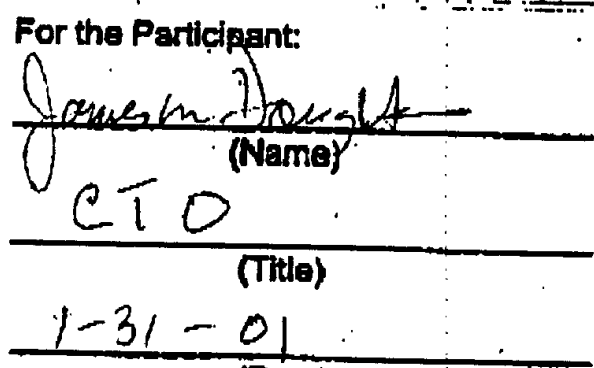

(Date) 